\title{
Peaks above the Harrison-Zel'dovich spectrum due to the Quark-Gluon to Hadron Transition
}

\author{
Christoph Schmid, Dominik J. Schwarz, and Peter Widerin* \\ Institut für Theoretische Physik, ETH-Hönggerberg, CH-8093 Zürich
}

(October 20, 2018)

\begin{abstract}
The quark-gluon to hadron transition affects the evolution of cosmological perturbations. If the phase transition is first order, the sound speed vanishes during the transition, and density perturbations fall freely. This distorts the primordial Harrison-Zel'dovich spectrum of density fluctuations below the Hubble scale at the transition. Peaks are produced, which grow at most linearly in wavenumber, both for the hadron-photon-lepton fluid and for cold dark matter. For cold dark matter which is kinetically decoupled well before the QCD transition clumps of masses below $10^{-10} M_{\odot}$ are produced.

98.80.Cq, 12.38.Mh, 95.35.+d
\end{abstract}

Typeset using REVTEX

*e-mail: chschmid, dschwarz, widerin, all @itp.phys.ethz.ch 
QCD makes a transition from a quark-gluon plasma at high temperatures to a hadron gas at low temperatures. Lattice QCD simulations give a transition temperature $T_{\star} \sim 150 \mathrm{MeV}$ and indicate a first-order phase transition for the physical values of the $\mathrm{u}, \mathrm{d}$,s-quark masses [1]. The relevance of the QCD transition for cosmology, especially for big-bang nucleosynthesis [2], has been discussed before, but the focus was on effects of bubble formation [3, 1]. In this paper we look at matter averaged over scales $\lambda$ much larger than the bubble separation. We show that for a first-order phase transition the sound speed $c_{s}=(\partial p / \partial \rho)_{s}^{1 / 2}$ drops to zero for these wavelengths when the transition temperature $T_{\star}$ is reached, stays zero for the entire time until the phase transition is completed, and afterwards suddenly rises back to $c_{s} \approx c / \sqrt{3}$. In contrast the pressure stays positive and varies continuously, although it goes below the radiation fluid value $p=\rho / 3$. Since $c_{s}$ is zero during the transition, there are no pressure perturbations, no pressure gradients, no restoring forces. Pre-existing cosmological perturbations, generated by inflation [5] with a Harrison-Zel'dovich spectrum [6], go into free fall. The superhorizon modes (at the time of the transition) remain unaffected. The subhorizon modes develop peaks in $\delta \rho / \rho$ which grow with wavenumber $k>k_{\star}$, where $k_{\star}^{\text {phys }} \sim$ Hubble rate $H$ at the end of the QCD transition. The details of this growth depend on the QCD equation of state near $T_{\star}$. We analyze two cases: First we use the bag model [7], which gives a simple parameterization and allows a simple discussion of the effects. It gives a maximal latent heat, and produces peaks in $\delta \rho / \rho$ which grow linearly in $k$. Next we use lattice QCD results [1,8,9], which indicate a smaller latent heat, and we fit $s / T^{3}=$ $C_{1}+C_{2}\left(1-T_{\star} / T\right)^{1 / 3}$ above $T_{\star}$. This produces peaks in $\delta \rho / \rho$ which grow as $k^{3 / 4}$.

The sound speed (for wavelength $\lambda$ much larger than the bubble separation), $c_{s}=$ $(\partial p / \partial \rho)_{s}^{1 / 2}$, must be zero during a first-order phase transition of a fluid with negligible chemical potential, since the fluid must obey

$$
\rho+p=T \frac{d p}{d T}
$$

according to the second law of thermodynamics. Because the energy density $\rho$ is discontinuous in temperature at $T_{\star}$ for a first-order phase transition, the pressure $p$ must be continuous with a discontinuous slope. As the universe expands at fixed temperature $T_{\star}$ during the phase transition, $\rho$ as a function of time slowly decreases from $\rho_{+}\left(T_{\star}\right)$ to $\rho_{-}\left(T_{\star}\right), p$ stays constant at $p\left(T_{\star}\right)$, and therefore $c_{s}$ is zero during the whole time of the phase transition.

The interaction rates in the QCD-photon-lepton fluid are much larger than the Hubble rate, $\Gamma / H \gg 1$, therefore we are very close to thermal and chemical equilibrium, the QCD transition is very close to a reversible thermodynamic transformation, and the entropy in a comoving volume is approximately conserved. Estimates show that supercooling, hence entropy production, is negligible, $\left(T_{\star}-T_{\text {supercooling }}\right) / T_{\star} \sim 10^{-3}$ [10]. Bubble formation during the QCD phase transition is unimportant for our analysis, estimates give a bubble separation $\ell_{b} \sim 1 \mathrm{~cm}$ [4], while the Hubble radius at the QCD transition is $R_{H} \sim 10 \mathrm{~km}$, therefore $\ell_{b} / R_{H} \sim 10^{-6}$. We shall analyze perturbations with $\lambda \gg \ell_{b}$.

In the bag model [7] it is assumed that for $T>T_{\star}$ the quark-gluon plasma (QGP) obeys

$$
p_{\mathrm{QGP}}(T)=p_{\mathrm{QGP}}^{\text {ideal }}(T)-B \text {, }
$$

where $p_{\mathrm{QGP}}^{\text {ideal }}(T)=\left(\pi^{2} / 90\right) g_{\mathrm{QGP}}^{*} T^{4}, g^{*}$ is the effective number of relativistic helicity states, and $B$ is the bag constant. We include u,d-quarks and gluons in the quark-gluon plasma, 
$\gamma, e, \mu$, and 3 neutrinos in the photon-lepton fluid $(\gamma \mathrm{L})$, and for $T<T_{\star}$ we have a hadron gas (HG) of pions. We treat the pions as massless and ideal, because their contribution is small anyway, $g_{\mathrm{HG}}^{*} / g_{\mathrm{QGP}}^{*}=3 / 37$ and $g_{\mathrm{HG}}^{*} / g_{\gamma \mathrm{L}}^{*}=3 / 14.25 . \rho$ follows from Eq. (2) via the second law of thermodynamics, Eq. (1) and $s$ from $s=d p / d T$. This gives for the quark-gluon plasma

$$
\begin{aligned}
& \rho_{\mathrm{QGP}}(T)=\rho_{\mathrm{QGP}}^{\text {ideal }}(T)+B \\
& s_{\mathrm{QGP}}(T)=s_{\mathrm{QGP}}^{\text {ideal }}(T) .
\end{aligned}
$$

The bag constant is determined by the critical temperature $T_{\star}$ via $p_{\mathrm{QGP}}\left(T_{\star}\right)=p_{\mathrm{HG}}\left(T_{\star}\right)$.

The latent heat, $L \equiv T_{\star} \Delta s$, should be compared with the difference in entropy between an ideal HG and an ideal QGP. This defines the ratio $R_{L} \equiv L /\left(T_{\star} \Delta s\right)^{\text {ideal }}$. The bag model gives $R_{L}=1$. Lattice QCD indicates that the transition is first order both for quenched QCD (no dynamical quarks) [8] and for QCD with three quarks and physical masses [1]. For the latter case neither the value of the latent heat nor the equation of state are available. Quenched QCD gives $L / T_{\star}^{4} \approx 1.4$ [9], which implies $R_{L} \approx 0.2$. We fit the shape of the QCD entropy to quenched QCD data [B] for $T>T_{\star}$ by

$$
\frac{s_{\mathrm{QGP}}^{\mathrm{fit}}}{s_{\mathrm{QGP}}^{\text {ideal }}}=1+\left[\left(1-\frac{T_{\star}}{T}\right)^{\gamma}-1\right] \frac{\Delta g^{*}}{g_{\mathrm{QGP}}^{*}}\left(1-R_{L}\right),
$$

where $\Delta g^{*} \equiv g_{\mathrm{QGP}}^{*}-g_{\mathrm{HG}}^{*}$ and $R_{L}=0.2$. A good fit for our purpose is obtained for $\gamma \in$ $(0.3,0.4)$. We fix $\gamma=1 / 3$.

The growth of the scale factor during the $c_{s}^{2}=0$ part of the QCD transition, $a_{+} / a_{-}$, follows from the conservation of entropy in a comoving volume,

$$
\frac{a_{+}}{a_{-}}=\left[1+R_{L} \frac{\Delta g^{*}}{g_{\mathrm{after}}^{*}}\right]^{\frac{1}{3}} \approx\left\{\begin{array}{ll}
1.4 & R_{L}=1 \\
1.1 & R_{L}=0.2
\end{array},\right.
$$

taking into account photons, leptons, and hadrons in $g_{\text {after }}^{*}$. Fig. 1 1 shows the evolution of the sound speed with the scale factor $a$. Above $T_{\star}$, the sound speed in the bag model has the value for an ultrarelativistic ideal gas, $c_{s}=1 / \sqrt{3}$, because the bag constant drops out when forming $d p / d T$ and $d \rho / d T$ in Eqs. (2) and (3). The sound speed vanishes for about a third of a Hubble time for $R_{L}=1$ and for a tenth of a Hubble time if $R_{L}=0.2$. The pressure does not drop all the way to zero, it drops to $p_{\gamma \mathrm{L}}\left(T_{\star}\right)+p_{\mathrm{HG}}\left(T_{\star}\right)$.

The evolution of linear cosmological perturbations through the QCD transition is analyzed in the longitudinal sector (density perturbations) for perfect fluids. We choose a slicing $\Sigma$ of space-time with unperturbed mean extrinsic curvature, $\delta\left[\operatorname{tr} K_{i j}(\Sigma)\right]=0$. This implies that our fundamental observers, which are defined to be at rest on the slice $\Sigma, \underline{u}($ obs $)=\underline{n}(\Sigma)$, have relative velocities, which in the mean over all directions follow an unperturbed Hubble flow. If the coordinate choice (gauge choice) is such that the time coordinate $t$ is constant on the slices $\Sigma$, the gauge is fixed to be the uniform expansion (Hubble) gauge [11]. As fundamental evolution equations for each fluid we have $\nabla_{\mu} T^{\mu \nu}=0$, i.e. the continuity equation and (in the longitudinal sector) the 3-divergence of the Euler equation of general relativity,

$$
\begin{aligned}
\partial_{t} \epsilon & =-3 H(\epsilon+\pi)-\triangle \psi-3 H(\rho+p) \alpha \\
\partial_{t} \psi & =-3 H \psi-\pi-(\rho+p) \alpha,
\end{aligned}
$$


where $\epsilon \equiv \delta \rho, \pi \equiv \delta p, \rho \equiv \rho_{0}, p \equiv p_{0}, \vec{\nabla} \psi \equiv \vec{S}=$ momentum density (Poynting vector), $\alpha=$ lapse function. The system of dynamical equations is closed by Einstein's $R_{\hat{0} \hat{0}}$-equation, the general relativistic version of Poisson's equation,

$$
(\triangle+3 \dot{H}) \alpha=4 \pi G(\epsilon+3 \pi),
$$

together with the equation of state. Equations (7) - (9) define our general relativistic Cauchy problem in linear perturbation theory in the longitudinal sector with initial data $(\epsilon, \psi)$ freely chosen on $\Sigma_{i}$. These three equations are the Jeans equations extended to general relativity in the longitudinal sector. In all three of them the mean over all directions is taken. This fact matches our slicing condition that in the mean over all directions the relative velocity of our fundamental observers is unperturbed. Therefore the uniform expansion (Hubble) gauge could be called the 'longitudinal Jeans gauge'.

It is convenient to work with the dimensionless variables $\delta \equiv \epsilon / \rho$ (density contrast), $\hat{\psi} \equiv k^{\text {phys }} \psi / \rho(\sim$ peculiar velocity $)$ and with conformal time, ()$^{\prime} \equiv \partial_{\eta} \equiv a \partial_{t}$. In our numerical analysis we have used the exact general relativistic equations, but it is instructive to look at the subhorizon approximation, $\lambda^{\text {phys }} \ll H^{-1}$, where one can drop $\dot{H}$ in the general relativistic Poisson equation (9) and the time dilation term (last term) in the continuity equation (7). Furthermore, if we take the limit in which the QCD transition time is much shorter than the Hubble time, $\left(t_{+}-t_{-}\right) \ll H^{-1}$, and if we integrate the equations during a correspondingly short time interval, we can drop the remaining terms proportional to $H$, and Eqs. (7) - (9) simplify to

$$
\begin{aligned}
\delta^{\prime} & =k \hat{\psi} \\
\hat{\psi}^{\prime} & =-c_{s}^{2} k \delta-(1+w) k \alpha \\
\left(\frac{k}{a H}\right)^{2} \alpha & =-\frac{3}{2}\left(1+3 c_{s}^{2}\right) \delta,
\end{aligned}
$$

where $w \equiv p / \rho$. With these approximations the evolution of a mode $k$ of cosmological perturbations can be solved analytically.

The origin of large peaks in $\delta \rho / \rho$ for $k \gg k_{\star}$, where $k_{\star}^{\text {phys }} \sim H$ at the transition, is easily understood in the bag model. For the dynamics of the radiation fluid (QCD, photons, leptons) one can neglect cold dark matter $(\mathrm{CDM})$ since $\rho_{\mathrm{CDM}} / \rho_{\mathrm{RAD}}=a / a_{\text {equality }} \approx 10^{-8}$. The radiation fluid in each mode makes standing acoustic oscillations before and after the QCD transition with gravity negligible and with equal amplitudes of $\delta$ and $\sqrt{3} \hat{\psi}$. The solution before the QCD transition is $\delta(\eta)=A_{\text {in }} \cos \left[\omega\left(\eta-\eta_{-}\right)-\varphi_{-}\right]$and $\hat{\psi}=\delta^{\prime} / k$, where $\omega=k c_{s}=k / \sqrt{3}$, and $\varphi_{-}$is the phase of the acoustic oscillation at $\eta_{-}$, i.e. at the beginning of the QCD transition. During the QCD transition the sound speed is zero, there are no restoring forces from pressure gradients, the radiation fluid goes into free fall. But during this free fall gravity is again negligible for the radiation fluid, if $\left(t_{+}-t_{-}\right) \ll H^{-1}$. This is inertial motion in the sense of Newton. The peculiar velocity is constant in time, $\hat{\psi}(\eta)=\hat{\psi}_{-}$, and the density contrast grows linearly in time with a slope $k, \delta(\eta)=\delta_{-}+k\left(\eta-\eta_{-}\right) \hat{\psi}_{-}$. Thus, the final amplitude $A_{+}$grows linearly in $k$ modulated by $\sin \left(\varphi_{-}\right)$, which produces peaks in the spectrum. The height of these peaks is

$$
\left.\frac{A_{+}}{A_{\text {in }}}\right|_{\text {peaks }}=\frac{\left(\eta_{+}-\eta_{-}\right) k}{\sqrt{3}} \equiv \frac{k}{k_{1}},
$$


for $k \gg k_{1}$. The usual free fall growing mode at subhorizon scales behaves totally different: $\hat{\psi} / \delta \sim H / k^{\text {phys }} \ll 1$ and $\delta_{+} / \delta_{-}=\left(\eta_{+} / \eta_{-}\right)^{2} \approx 2$. In our case the initial peculiar velocity $\hat{\psi}$ from the acoustic oscillations of the radiation fluid is enormously larger than in the usual free fall growing mode for $k \gg k_{\star}$. COBE observations [12 normalize the subhorizon spectrum of density perturbations as $(\delta \rho / \rho)_{\lambda} \sim 10^{-4}$, if there is no tilt in the spectrum. Hence in the bag model radiation-fluid modes with $k / k_{1} \gtrsim 10^{4}$ go nonlinear by the end of the QCD transition.

Our numerical results for the spectrum of density perturbations from the lattice QCD fit Eq. (5) are given in Fig. 2. We show the enhancement of the amplitude $A_{\mathrm{RAD}} \equiv\left(\delta_{\mathrm{RAD}}^{2}+\right.$ $\left.3 \hat{\psi}_{\mathrm{RAD}}^{2}\right)^{1 / 2}$ of the acoustic oscillations of the radiation fluid after the transition compared to the amplitude without transition. For CDM we show the amplitude $A_{\mathrm{CDM}} \equiv\left|\delta_{\mathrm{CDM}}\right|$ at $T_{\star} / 10$ compared to $A_{\mathrm{RAD}}$ without transition. In both cases we obtain peaks over the Harrison-Zel'dovich spectrum of primordial adiabatic density fluctuations. The modes $k$ (horizontal axis) are labeled by the CDM mass contained in a sphere of radius $\lambda / 2=\pi / k$. The positions of the first few peaks and dips are the same for the lattice QCD fit and for the bag model. In both cases the beginning of the peak-dip structure is at $k_{1}$, see Eq. (11), which is $\sim k_{\star}$. $k_{1}$ corresponds to $M_{1}^{\mathrm{CDM}} \approx 9 \times 10^{-9} M_{\odot}$. The peaks grow as $\left(k / k_{2}\right)^{3 / 4}$ for $k \gg k_{2}$, see Eq. (13). The radiation energy inside $\lambda_{1} / 2$ is $\sim 1 M_{\odot}$, but it gets redshifted as $M_{\mathrm{RAD}}(a) \sim\left(a_{\text {equality }} / a\right) M_{\mathrm{CDM}}$. The time evolution of a subhorizon mode is shown in Fig. [3. During $c_{s}=0 \delta_{\mathrm{RAD}}$ grows linearly as in the bag model. Above $T_{\star}$ the evolution differs because $c_{s}<1 / \sqrt{3}$.

The slower rise in $k$ for the lattice QCD fit can be understood by a WKB analysis. With the same approximations as above, Eqs. (10) reduce to

$$
\delta^{\prime \prime}+c_{s}^{2} k^{2} \delta=0 .
$$

Under the WKB condition, $\left|d c_{s} / d \eta\right| / c_{s} \ll \omega=c_{s} k$, the solution reads $\delta=$ $A_{\text {in }}\left(3 c_{s}^{2}\right)^{-1 / 4} \cos \left(k \int c_{s} d \eta\right)$. Note that the peculiar velocity $(\sim \hat{\psi})$ decreases with $c_{s}^{1 / 2}$, which can be seen in Fig. [3. Just above $T_{\star}$ the sound speed may be approximated by $c_{s} \propto\left(\eta_{-}-\eta\right)$. This gives the solutions of Eq. (12) as $\delta \propto z^{1 / 4} J_{ \pm 1 / 4}(z)$ with $z \equiv c_{s} k\left(\eta_{-}-\eta\right) / 2$. The normalization for large $k$ is provided by the WKB solution. At $\eta_{-}$one matches the linearly growing solution in the regime of vanishing sound speed $\left(\eta_{-}<\eta<\eta_{+}\right)$. The final amplification is

$$
\left.\frac{A_{+}}{A_{\text {in }}}\right|_{\text {peaks }}=\frac{\sqrt{\pi}}{2 \Gamma\left(\frac{5}{4}\right)}\left|\frac{c_{s}^{\prime}\left(\eta_{-}\right)}{3 k}\right|^{\frac{1}{4}}\left(\eta_{+}-\eta_{-}\right) k \equiv\left(\frac{k}{k_{2}}\right)^{\frac{3}{4}},
$$

for $k \gg k_{2} . k_{2}$ depends on the duration of the $c_{s}=0$ regime and thus depends on $R_{L}$. For $R_{L}=0.2$ it corresponds to a mass $M_{2}=2 \times 10^{-10} M_{\odot}$, which is indicated in Fig. 2 together with the asymptotic envelope $\left(k / k_{2}\right)^{3 / 4}$. Without tilt COBE normalized modes with $k / k_{2} \gtrsim 10^{5}$ go nonlinear by the end of the QCD transition. The perturbations in the radiation fluid will get wiped out by collisional damping from neutrinos at temperatures below $T_{\star}$ but above $1 \mathrm{MeV}$.

For cold dark matter we consider any non-relativistic matter which decouples kinetically well before the QCD transition. The neutralino, most likely the lightest supersymmetric particle [13], is weakly interacting. Thus it decouples kinetically around $T \sim 1 \mathrm{MeV}$ and is excluded to make our CDM. It would belong to the radiation fluid at $T_{\star}$. Candidates for our CDM are axions or primordial black holes. 
CDM falls into the gravity wells generated during the transition by the radiation fluid. In the bag model this leads to peaks in CDM which grow linearly for $k \gg k_{1}$, i.e. $\delta_{+}^{\mathrm{CDM}}-\delta_{-}^{\mathrm{CDM}}=$ $\left[H\left(t_{+}-t_{-}\right) / 2\right]^{2} \delta_{+}^{\mathrm{RAD}}$. After the transition $\delta^{\mathrm{CDM}}$ grows logarithmically. For our lattice QCD fit the CDM peaks are shown in Fig. 2. An analytic analysis of the CDM evolution will be presented in a longer paper.

The implications of these peaks above the Harrison-Zel'dovich spectrum generated in a standard scenario with a first order QCD transition are:

1) For CDM which is kinetically decoupled well before the QCD transition clumps with $M_{\mathrm{CDM}} \lesssim 10^{-10} M_{\odot}$ are produced. They go nonlinear after equality and virialize by violent gravitational relaxation. Assuming a COBE normalized spectrum with tilt $n-1=0(0.2)$ and $3 \sigma$ peaks the size of $10^{-10} M_{\odot}$ clumps is $\approx 14 \mathrm{AU}(1 \mathrm{AU})$.

2) Big-Bang Nucleosynthesis will not be affected by nonlinear acoustic oscillations of the radiation fluid for $k / k_{1} \gtrsim 10^{4}$ (bag model) resp. $10^{6}$ (lattice QCD fit), because they are wiped out by collisional damping from neutrinos before Big-Bang Nucleosynthesis.

3) Primordial black hole formation is unlikely, because the nonlinear acoustic oscillations in the radiation fluid are generated far below the Hubble scale at the QCD transition. This is in contrast to Ref. [14.

We like to thank J. A. Bardeen, P. Jetzer, F. Karsch, V. Mukhanov, J. Silk, and N. Straumann for helpful discussions. C. S. thanks the Center for Particle Astrophysics in Berkeley and the Fermilab Astrophysics Center for hospitality. D. S. and P. W. thank the Swiss National Science Foundation for financial support. 


\section{REFERENCES}

[1] C. DeTar, Nucl. Phys. B (Proc. Suppl.) 42, 73 (1995); T. Blum et al., Phys. Rev. D 51, 5153 (1995); Y. Iwasaki et al., Z. Phys. C 71, 343 (1996).

[2] J. H. Applegate and C. J. Hogan, Phys. Rev. D 31, 3037 (1985); J. H. Applegate, C. J. Hogan, and R. J. Scherrer, Phys. Rev. D 35, 1151 (1987); G. M. Fuller, G. J. Mathews, and C. R. Alcock, Phys. Rev. D 37, 1380 (1988); R. A. Malaney and G. J. Mathews, Phys. Rep. 229, 145 (1993).

[3] E. Witten, Phys. Rev. D 30, 272 (1984); K. Kajantie and H. Kurki-Suonio, Phys. Rev. D 34, 1719 (1986); L. P. Csernai and J. I. Kapusta, Phys. Rev. D 46, 1379 (1992); J. Ignatius et al., Phys. Rev. D 50, 3738 (1994).

[4] A. A. Coley and T. Trappenberg, Phys. Rev. D 50, 4881 (1994); M. B. Christiansen and J. Madsen, Phys. Rev. D 53, 5446 (1996).

[5] V. Mukhanov and G. Chibisov, Pis'ma Zh. Eksp. Teor. Fiz. 33, 549 (1981); A. Starobinsky, Phys. Lett. B 117, 175 (1982); A. Guth and S.-Y. Pi, Phys. Rev. Lett. 49, 1110 (1982); S. Hawking, Phys. Lett. B 115, 295 (1982); J. A. Bardeen, P. Steinhardt and M. Turner, Phys. Rev. D 28, 679 (1983).

[6] E. R. Harrison, Phys. Rev. D 1, 2726 (1970); Ya. B. Zel'dovich, Mon. Not. R. Astr. Soc. 160 1P (1972).

[7] T. DeGrand and K. Kajantie, Phys. Lett. 147B, 273 (1984).

[8] G. Boyd et al., Phys. Rev. Lett. 75, 4169 (1995); Nucl. Phys. B469, 419 (1996).

[9] Y. Iwasaki et al., Phys. Rev. D 46, 4657 (1992); 49, 3540 (1994); B. Beinlich, F. Karsch, and A. Peikert, preprint hep-lat/9608141 (1996).

[10] Based on G. M. Fuller, G. J. Mathews, and C. R. Alcock, Phys. Rev. D 37, 1380 (1988).

[11] J. A. Bardeen, Phys. Rev. D 22, 1882 (1980); and in Particle Physics and Cosmology, ed. A. Zee (Gordon and Breach, New York, 1989).

[12] G. Hinshaw et al., Astrophys. J. 464, L17 (1996).

[13] G. Jungman, M. Kamionkowski, and K. Griest, Phys. Rep. 267, 195 (1996).

[14] K. Jedamzik, preprint astro-ph/9605152 (1996). 


\section{FIGURES}

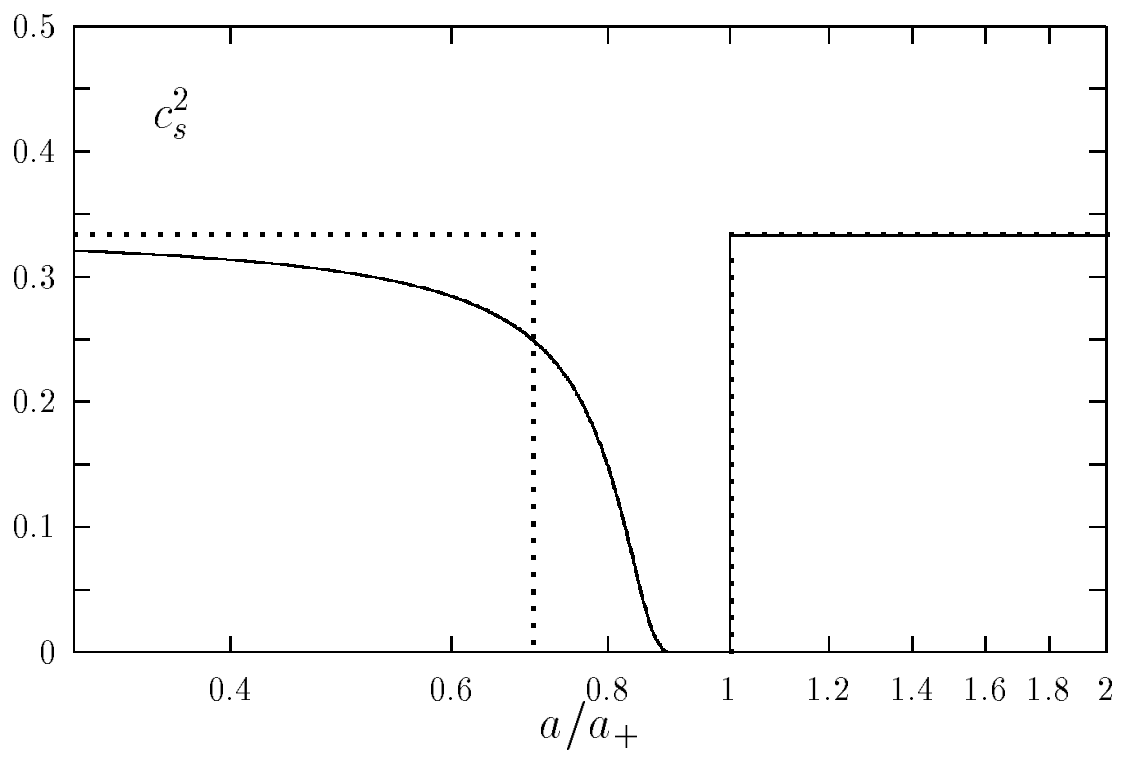

FIG. 1. The sound speed $c_{s}^{2}=(\partial p / \partial \rho)_{s}$ during the QCD transition for the bag model (dotted line) and for the lattice QCD fit (full line).

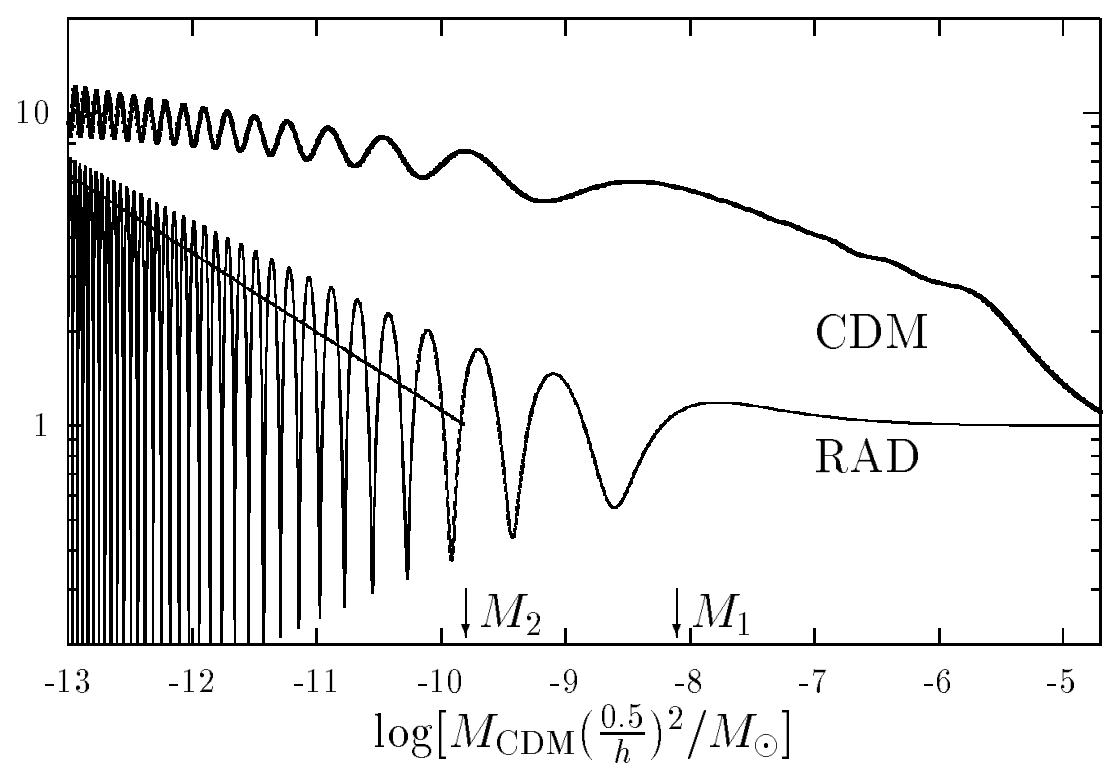

FIG. 2. The modifications of the CDM density contrast $A_{\mathrm{CDM}} \equiv\left|\delta_{\mathrm{CDM}}\right|\left(T_{\star} / 10\right)$ and of the radiation fluid amplitude $A_{\mathrm{RAD}} \equiv\left(\delta_{\mathrm{RAD}}^{2}+3 \hat{\psi}_{\mathrm{RAD}}^{2}\right)^{1 / 2}$ due to the QCD transition (lattice QCD fit). Both quantities are normalized to the pure Harrison-Zel'dovich radiation amplitude. On the horizontal axis the wavenumber $k$ is represented by the CDM mass contained in a sphere of radius $\pi / k$. 


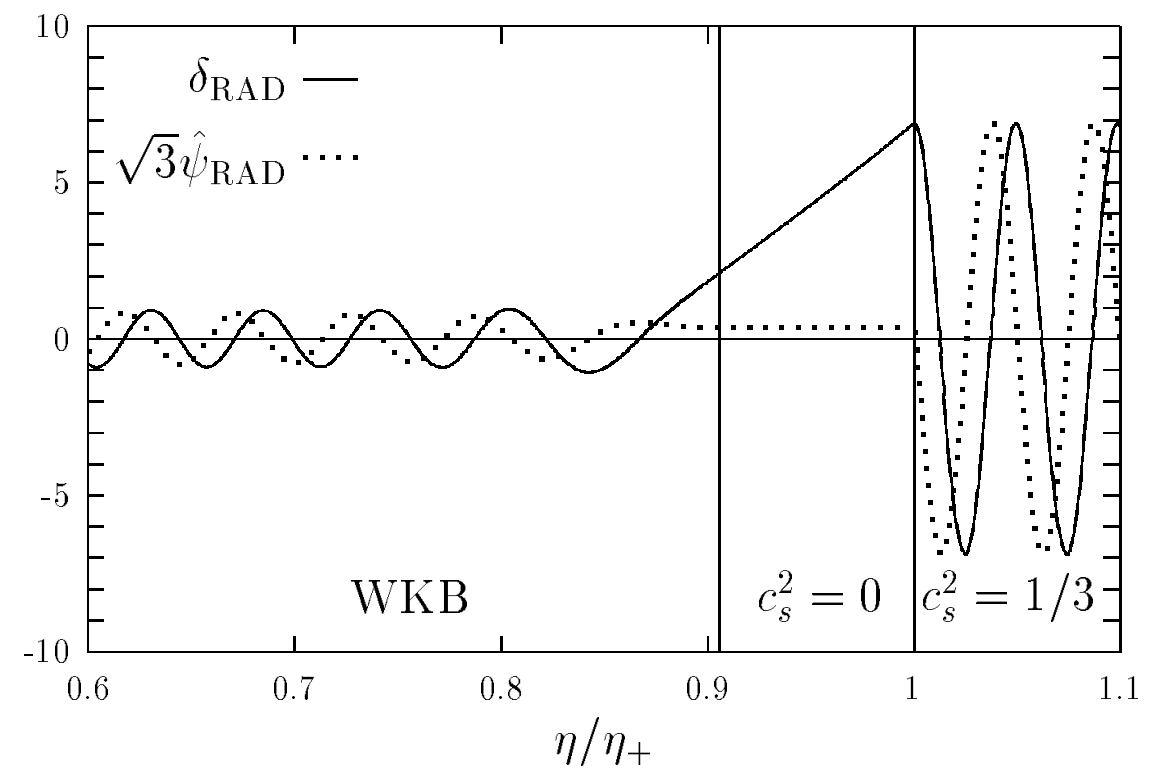

FIG. 3. The evolution in conformal time $\eta$ of the density contrast $\left(\delta_{\mathrm{RAD}}\right)$ and the peculiar velocity $\left(\sim \hat{\psi}_{\mathrm{RAD}}\right)$ of the radiation fluid for the highest peak of Fig. 2 in uniform expansion (Hubble) gauge. During the QCD transition in the lattice QCD fit — marked by the 2 vertical lines — the velocity stays approximately constant and the density contrast grows linearly. The amplitude in the WKB regime is normalized to 1 long before the transition. 